\title{
Increase of orexin $A$ in the peripheral blood of adolescents with Internet gaming disorder
}

\author{
MI RAN CHOI, HYUN CHO, JI-WON CHUN, JAE HYUN YOO and DAI-JIN KIM*
}

Department of Psychiatry, Seoul St. Mary's Hospital, The Catholic University of Korea College of Medicine, Seoul, Republic of Korea

(Received: May 28, 2019; revised manuscript received: September 10, 2019; second revised manuscript received: October 16, 2019; accepted: November 24, 2019)

\begin{abstract}
Background and aims: Overindulgence in Internet gaming, which is related to rapid development of the online game industry, can cause a psychiatric disorder known as Internet gaming disorder (IGD). The number of adolescents with IGD is on the rise in countries with developed Internet technologies, such as South Korea. Therefore, it is important to develop biomarkers to detect patients at high risk of IGD. This study investigated expression levels of proteins in the blood of adolescents to provide insight into the development of biomarkers. Methods: We collected blood samples from 73 subjects [ 40 healthy adolescents (Internet gaming control, IGC) and 33 adolescents with IGD] between 13:00 and 15:00. We analyzed the expression levels of orexin A, oxytocin, cortisol, melatonin, BDNF, sICAM-1, RANTES, and NCAM using multiplex assay kits. Results: Orexin A was significantly $(p=.016)$ elevated in the IGD group and the expression levels of melatonin tended to be higher $(p=.055)$ in the IGD group. On the other hand, increased Internet gaming time in the IGD group was negatively correlated $(p=.041)$ with expression of BDNF. On the contrary, sICAM-1 associated with inflammation exhibited the tendency of the positive correlation $(p=.073)$ with Internet gaming time in the IGD group. Discussion and conclusions: We identified elevation of orexin A in the peripheral blood of adolescents with IGD and a negative correlation between Internet gaming time and BDNF in adolescents with IGD. Our results provide useful information to understand the pathophysiology of IGD in adolescents.
\end{abstract}

Keywords: adolescent, BDNF, Internet gaming disorder, melatonin, orexin A

\section{INTRODUCTION}

As the Internet continues to develop rapidly, the online gaming industry also continues to grow. As Internet games become popular, overindulgence in Internet games may cause social problems, such as increased crime and significant impairments at school and work (Widyanto \& Griffiths, 2006; Young, 2004). Overuse of Internet games also causes depression, anxiety disorders, and cognitive impairments and is considered a psychiatric disease termed Internet gaming disorder (IGD; Aydm \& San, 2011; Dalbudak et al., 2013; Kuss, Griffiths, Karila, \& Billieux, 2014; Wang, Cho, \& Kim, 2018). Overuse of Internet gaming by adolescents is prevalent in countries with developed Internet technologies, including South Korea (Kuss et al., 2014; Moreno, Jelenchick, Cox, Young, \& Christakis, 2011; Müller, Glaesmer, Brähler, Woelfling, \& Beutel, 2014). As a result, adolescents in these countries are more likely to be exposed to circumstances that can lead to IGD. IGD is currently described in Section III of the fifth edition of the Diagnostic and Statistical Manual of Mental Disorders (DSM-5; American Psychiatric Association, 2013). In addition, the World Health Organization (WHO, 2018) added gaming disorder, including digital or video gaming, to the addictive disorders section of its new International Classification of Diseases (ICD-11) in 2018.
Adolescence is a period in which important maturational rearrangements occur in hormonal and brain systems (Izenwasser, 2005). Studies have shown that adolescents with IGD exhibit low functional connectivity in the brain (Chun, Choi, Cho, Lee, \& Kim, 2015; Du et al., 2017; Kang, Jung, Park, \& Han, 2018; Lubman, Cheetham, \& Yucel, 2015; Qi et al., 2015), so overexposure to Internet gaming during this period may negatively affect neurotransmitter systems, structure and functional connectivity in the brain, as well as the expression of hormones and neuropeptides in the peripheral blood. Furthermore, these negative effects could induce emotional and behavioral changes that continue into adulthood. Recently, some studies have found a strong correlation between Internet game addiction and psychological problems including depression and anxiety in adolescents with IGD (Boyer \& Dickerson, 2003; Kuss \& Griffiths, 2012; Torres-Rodriguez, Griffiths, Carbonell, \& Oberst, 2018). Neuroimaging analysis has revealed that adolescents with IGD exhibit not only alteration of gray matter volume (Pan et al., 2018), but also reduced activation

\footnotetext{
* Corresponding author: Dai-Jin Kim, MD, PhD; Department of Psychiatry, Seoul St. Mary's Hospital, The Catholic University of Korea College of Medicine, 222 Banpo-daero, Seocho-gu, Seoul 06591, Republic of Korea; Phone: +82 22258 6086; Fax: +82 2 594 3870; E-mail: kdj922@catholic.ac.kr
}

This is an open-access article distributed under the terms of the Creative Commons Attribution-NonCommercial 4.0 International License, which permits unrestricted use, distribution, and reproduction in any medium for non-commercial purposes, provided the original author and source are credited, a link to the CC License is provided, and changes - if any - are indicated. 
of the orbitofrontal cortex associated with cognitive control (Chun et al., 2015), providing additional evidence that Internet game addiction may impact the structure and function of the brain. Another study found that polymorphisms related to dopamine genes are associated with excessive Internet game play in adolescents, suggesting that genetic background may also be a risk factor for occurrence of IGD in adolescents (Han et al., 2007). Recently, our team documented that three micro RNAs (hsa-miR-200c-3p, hsamiR-26b-5p, and hsa-miR-652-39), which serve as epigenetic regulators, are downregulated in adolescents with IGD, providing insight into the pathophysiology of IGD (Lee et al., 2018). Although IGD in adolescents has very important clinical and social aspects, and attempts to develop biomarkers for IGD are progressing gradually, the pathophysiology of IGD is poorly understood and there is still no definitive biomarker for the diagnosis or treatment of IGD.

Orexin A, a neuropeptide that originates from the hypothalamus and perifornical area, is involved in appetite regulation (Sakurai et al., 1998) and mediation of diverse behaviors including drug-seeking, stress, depression, anxiety, and eating (Bonnavion, Jackson, Carter, \& de Lecea, 2015; Connor et al., 2017; Sweet, Levine, Billington, \& Kotz, 1999). According to previous studies, activation of the lateral hypothalamus orexin neurons reinstates drug-seeking behavior such as conditioned preference related to cocaine or morphine and orexin knockout in rodents decreases cocaine-seeking behaviors (Harris, Wimmer, \& Aston-Jones, 2005; Steiner et al., 2018). These studies suggest that the orexin system may be responsible for driving the motivation and reward circuits associated with drug addiction. Based on the observation that the orexin system is associated with drug addiction, investigation is needed to ascertain if this system is also correlated with behavioral addictions like IGD. Oxytocin is a neurohormone that is produced in the hypothalamus and affects learning, memory, reward, and stress (Lee, Rohn, Tanda, \& Leggio, 2016). Previous studies have found that short-term use of cocaine increases oxytocin (Johns, Caldwell, \& Pedersen, 1993), but long-term use of cocaine decreases oxytocin (Sarnyai \& Kovacs, 1994) in the rat hippocampus. Another study found that levels of oxytocin in the blood decreased in alcoholics, as well as a group with acute alcohol administration (Lynn, 1970; Marchesi, Chiodera, Brusamonti, Volpi, \& Coiro, 1997). Overall, these findings suggest that changes in the expression of oxytocin may vary according to the drug or situation.

Melatonin is known to control pleiotropic signaling pathways and biological rhythms (Onaolapo \& Onaolapo, 2018). Modulation of melatonin has been reported to change behaviors and physiological functions in substance addiction, including drug and alcohol addiction (Takahashi, Vengeliene, \& Spanagel, 2017; Vengeliene, Noori, \& Spanagel, 2015). In contrast, a recent in vitro study revealed that melatonin has a protective effect on normal cells but induces apoptosis in tumoral trophoblast cells, indicating that the function of melatonin can vary according to circumstances (Sagrillo-Fagundes, Bienvenue-Pariseault, \& Vaillancourt, 2019). Cortisol, a steroid hormone, is released by activation of the hypothalamic-pituitary-adrenal axis in response to stress or hypoglycemia (Yiallouris et al., 2019). Previous studies have reported that cortisol levels are elevated in the saliva of pathologic online game users and in the blood of adolescents with IGD, suggesting that cortisol secretion may be associated with Internet gaming addiction (Hebert, Beland, Dionne-Fournelle, Crete, \& Lupien, 2005; Kim \& Kim, 2013; Kim et al., 2019). However, there is not yet enough evidence that the aforementioned proteins are associated with addiction, so it is important to investigate whether these proteins are related to behavioral addiction such as IGD.

Brain-derived neurotrophic factor (BDNF) regulates neurotransmitter release, neurogenesis, synaptic plasticity, as well as axonal and dendritic morphology (Chao, 2003; Kowianski et al., 2018). In addition, BDNF has been reported to be associated with the development and maintenance of addictive disorders (Corominas, Roncero, Ribases, Castells, \& Casas, 2007). It has been reported that BDNF expression is changed in substance addiction including alcohol and drug addictions (Fumagalli, Di Pasquale, Caffino, Racagni, \& Riva, 2007; Garcia-Marchena et al., 2017; Xu et al., 2019). According to a systematic review and meta-analysis by Ornell et al. (2018), the expression levels of serum BDNF are reduced in active drug users (e.g., alcohol and cocaine). Conversely to substance addiction, pathological gambling disorder (PGD), a behavioral addiction, has been associated with an increase in serum BDNF expression (Angelucci et al., 2013; Choi et al., 2016; Geisel, Banas, Hellweg, \& Muller, 2012). A recent pilot study investigating BDNF serum levels in male patients with IGD and normal male controls showed that BDNF expression levels did not differ between the IGD and control groups (Geisel, Banas, Schneider, Hellweg, \& Muller, 2013), showing the necessity for further investigation of the association between BDNF expression and IGD. Soluble intracellular adhesion molecule-1 (sICAM-1), a circulating form of membrane-bound ICAM-1, has been reported to be elevated in inflammatory neurological diseases and myocardial infarction (Baraczka et al., 2001; Kraus et al., 1998; Ridker, Hennekens, Roitman-Johnson, Stampfer, \& Allen, 1998). Individuals using alcohol or nicotine show chronically elevated sICAM-1 in the blood (Bergmann, Siekmeier, Mix, \& Jaross, 1998; Bermudez, Rifai, Buring, Manson, \& Ridker, 2002; Sacanella et al., 1999). Therefore, expression changes of sICAM-1 may signal inflammatory circumstances vulnerable to impairments in the brain and other tissues. RANTES, also known as CC chemokine ligand 5, is a chemoattractant for inflammatory cells (Veillard et al., 2004), and upregulation of RANTES is associated with inflammation in various tissues (Mikolajczyk et al., 2016; Mohs et al., 2017; Zhou et al., 2018). Studies have found that RANTES is elevated in individuals with substance addiction including alcohol and cocaine (O'Halloran et al., 2016; Pereira et al., 2011), indicating that RANTES could be a potential biomarker for diagnosis of substance addiction. Neural cell adhesion molecule (NCAM) is associated with various mechanisms such as cell adhesion, neurite outgrowth, migration, and synaptic plasticity. Recent studies have reported that expression levels of the active form of NCAM (polysalicylated NCAM) or NCAM protein are changed in people with alcohol and drug addiction such as morphine and cocaine (Barker, Torregrossa, \& Taylor, 2012; Cao, Wang, Li, \& Zhang, 2016; Koob, 2003). Thus, 
changes of BDNF, sICAM-1, RANTES, and NCAM are associated with neurological diseases, particularly substance addiction. To understand the neuropathological mechanisms associated with IGD, it is important to assess whether expression changes of BDNF, sICAM-1, RANTES, and NCAM are related to Internet gaming addiction.

On the basis of these findings, we hypothesized that expression changes in neuropeptides (orexin A and oxytocin), hormones (melatonin and cortisol), and proteins (BDNF, sICAM-1, RANTES, and NCAM) related to neurological diseases may play a crucial role in the pathophysiology of IGD. The belief that expression changes in these factors in the blood indirectly represent their alterations in hormonal and brain systems suggests that these alterations affect development of IGD. In this study, we evaluated expression levels of eight proteins in the blood of adolescents with IGD and adolescents classified as Internet gaming controls (IGCs). We identified increased orexin A and a negative correlation between BDNF and Internet gaming time in the blood of the IGD group, suggesting their potential as biomarkers of IGD.

\section{METHODS}

\section{Participants}

We collected data from 1,700 youth surveys through a research company (Panel Marketing Interactive Co., Ltd., Seoul, Korea). In addition, a survey was conducted in 1,485 students from a single secondary school in Seoul. We included a notice about this study in the questionnaires. If students were willing to participate in the study, they were asked to provide personal and parent contacts. A total of 446 respondents expressed their intention to participate in this study. When we contacted them to explain the experiment, obtain parental consent, and to schedule them for the experiment, there were 369 whose parents refused to agree to participate in the experiment or were not contacted. Thus, 77 individuals (57 males and 19 females) provided informed consent for this study. All participants underwent a structured interview by a qualified clinical psychologist based on the Korean Kiddie Schedule for Affective Disorders and Schizophrenia - Present and Lifetime. To evaluate IGD, the participants underwent an additional interview by the clinical psychologist related to patterns of Internet gaming usage and problematic usage of Internet gaming according to the DSM-5 criteria. Among the 77 individuals, 4 were excluded according to the exclusion criteria. Exclusion criteria included past or current major medical disorders (e.g., diabetes mellitus), neurological disorders (e.g., seizure disorders and head injury), psychiatric disorders (e.g., major depressive disorder and anxiety disorders), mental retardation, or any substance abuse (e.g., tobacco, cannabis, and alcohol). Finally, 73 individuals [40 healthy adolescents (IGC) and 33 adolescents with IGD] were enrolled in this study. The general characteristics of the study subjects are summarized in Table 1.

\section{Measurement}

Demographic information. Participants were asked to state their sex, age, parent education years, socioeconomic status, and weekly online gameplay time.

IGD Scale. To determine the degree of the problematic use of Internet games through self-report, the nine "yes or no" items proposed by Petry et al. (2014) were used. Petry et al. (2014) composed nine items based on nine diagnostic criteria for diagnosis of IGD proposed in the DSM-5 and this has been translated into French, German, Dutch, Chinese, Japanese, etc., according to WHO's recommended translation procedure based on English-language questions. In this study, the Korean translation was used and Cronbach's $\alpha$ was .75 .

Perceived Stress Scale. The scale developed by Cohen, Kamarck, and Mermelstein (1983) was used to assess stress level. The scale was translated into Korean by Park and Seo (2010) and consists of questions that assess the degree to which people perceive their lives as stressful. Subjects indicate how often they have found their lives unpredictable,

Table 1. Demographic and clinical characteristics of participants

\begin{tabular}{lccc}
\hline Characteristic & IGC $(n=40)$ & IGD $(n=33)$ & $p$ \\
\hline Age (years) & $15.08 \pm 0.24$ & $15.18 \pm 0.31$ & .782 \\
Sex & $75 \%(n=30)$ & $78.8 \%(n=26)$ & .703 \\
$\quad$ Male & $25 \%(n=10)$ & $21.2 \%(n=7)$ & .247 \\
$\quad$ Female & $15.21 \pm 2.43$ & $14.50 \pm 2.56$ & .279 \\
Total education years of parents & $14.59 \pm 2.07$ & $13.91 \pm 2.99$ & .224 \\
$\quad$ Father & & & 14 \\
$\quad$ Mother & 23 & 14 & 5 \\
Socioeconomic status & 14 & $4.35 \pm 2.12$ & $.000^{* * *}$ \\
$\quad$ High & 2 & $28.31 \pm 0.76$ & $.000^{* * *}$ \\
Middle & $2.10 \pm 1.49$ & $3.46 \pm 0.26$ & $.000^{* * *}$ \\
$\quad$ Low & $23.48 \pm 0.96$ & & \\
Daily Internet gaming hours (hr) & $1.17 \pm 0.24$ & & \\
Stress & & & \\
IGD Scale &
\end{tabular}

Note. IGC: Internet gaming control; IGD: Internet gaming disorder.

$* * *$ Significant at $p<.001$. 
uncontrollable, and overloaded in the past month. The scale contains a total of 14 Likert-type 5-point items. The higher the total score, the higher the perceived stress level. In this study, Cronbach's $\alpha$ was .71.

\section{Blood collection and immunoassay}

Blood samples were collected from all participants between 13:00 and 15:00. The samples were kept at room temperature (RT) for $2 \mathrm{hr}$ to ensure sufficient coagulation of blood and centrifuged at $1,000 \times g$ for $10 \mathrm{~min}$. The upper phase containing serum was transferred into a fresh tube and stored at $-80{ }^{\circ} \mathrm{C}$ until used for immunoassay. Eight factors related to inflammation and neurobiology (orexin A, oxytocin, cortisol, RANTES, BDNF, melatonin, sICAM-1, and NCAM) were analyzed by using bead-based multiplex assay kits. This multiplex assay is advantageous in that it is able to analyze multiple proteins simultaneously and requires a small number of samples. Secretion levels of orexin A and oxytocin were analyzed with the Human Neuropeptide Magnetic Bead Panel (HNCSMAG-35K, EMD Millipore, Billerica, MA, USA) according to the manufacturer's instructions. To extract serum samples, we performed acetonitrile precipitation as follows. After adding $375 \mu \mathrm{l}$ of acetonitrile into $250 \mu \mathrm{l}$ of sample, the mixture was incubated at RT for $10 \mathrm{~min}$. After the mixture was centrifuged at $17,000 \times g$ for $5 \mathrm{~min}, 500 \mu \mathrm{l}$ of supernatant was moved into a 96-well collecting plate. The samples were vacuumdried and reconstituted with $200 \mu \mathrm{l}$ of assay for use in an immunoassay. The immunoassay procedure was as follows. An amount of $200 \mu \mathrm{l}$ of assay buffer was added into each plate well and incubated at RT for $10 \mathrm{~min}$. After removing the assay buffer, $50 \mu \mathrm{l}$ of standard and $50 \mu \mathrm{l}$ of assay buffer were added to the standard and sample wells, respectively. An amount of $50 \mu \mathrm{l}$ of the appropriate matrix solution was added to background and standard wells and $50 \mu \mathrm{l}$ of extracted serum samples were added to sample wells. A portion of $25 \mu \mathrm{l}$ of primary antibodies was added to each well and the plate was incubated at RT for $2 \mathrm{hr}$. After adding $25 \mu \mathrm{l}$ of beads to each well, the plate was incubated overnight at $4{ }^{\circ} \mathrm{C}$. The background, standards, and sample wells were washed with $200 \mu \mathrm{l}$ of wash buffer and $50 \mu \mathrm{l}$ of detection antibodies were added to each well. After incubating at RT for $1 \mathrm{hr}, 50 \mu \mathrm{l}$ of streptavidin-phycoerythrin was added to each well. The plate was incubated at RT for $30 \mathrm{~min}$. After washing with $200 \mu \mathrm{l}$ of wash buffer, $150 \mu \mathrm{l}$ of sheath fluid was added to each well, and the plate was read on a Luminex 200TM (EMD Millipore). Secretion levels of melatonin and cortisol were analyzed with the Human Circadian/Stress Magnetic Bead Panel (HNDG3MAG-35K, EMD Millipore). The serum sample extraction procedure was performed in the same manner as the acetonitrile precipitation method used in the analysis of orexin A and oxytocin. The extracted serum samples were diluted 1:4 with assay buffer prior to use in the immunoassay. The immunoassay procedure to measure secretion levels of melatonin and cortisol was performed in the same manner as the immunoassay used to measure secretion levels of orexin A and oxytocin except for application of diluted (1:4) extracted samples. Secretion levels of BDNF, sICAM-1, RANTES, and NCAM were analyzed with Human Neurodegenerative
Disease Magnetic Bead Panel 3 (HNDG3MAG-36K, EMD Millipore). Before the immunoassay, serum samples were diluted 1:100 in the assay buffer. An amount of $200 \mu \mathrm{l}$ of assay buffer was added into each well of the plate and incubated at RT for $10 \mathrm{~min}$. After removing the assay buffer, $25 \mu \mathrm{l}$ of standard and $25 \mu \mathrm{l}$ of assay buffer were added to standard and sample wells, respectively. About $25 \mu \mathrm{l}$ of the appropriate matrix solution was added to background and standard wells and $25 \mu \mathrm{l}$ of diluted (1:4) serum samples were added to sample wells. An amount of $25 \mu \mathrm{l}$ of beads was added to each well and the plate was incubated overnight at $4{ }^{\circ} \mathrm{C}$. The background, standards, and sample wells were washed with $200 \mu \mathrm{l}$ of wash buffer and $25 \mu \mathrm{l}$ of detection antibodies were added to each well. After incubating at RT for $1 \mathrm{hr}, 25 \mu \mathrm{l}$ of streptavidin-phycoerythrin was added to each well. The plate was incubated at RT for $30 \mathrm{~min}$. After washing with $200 \mu \mathrm{l}$ of wash buffer, $100 \mu \mathrm{l}$ of sheath fluid was added to each well, and the plate was read on the Luminex 200TM (EMD Millipore).

\section{Statistical analyses}

Statistical analyses were conducted with SPSS 18.0 software (SPSS Inc., Chicago, IL, USA) and Graphpad Prism 8 software (San Diego, CA, USA). Comparison of demographic and clinical variables and eight protein expression levels between the IGC and IGD groups was performed using two-sample $t$-tests and Mann-Whitney $U$ tests for normally distributed variables and non-parametric variables, respectively. The Kolmogorov-Smirnov test was performed to evaluate whether the variables and protein expression levels were normally distributed. The data were represented as mean \pm standard deviation for the $t$-test and as median and interquartile distance for the Mann-Whitney $U$ test. Correlations between expression levels of each protein and daily Internet gaming hours within each group were analyzed by Spearman's correlation coefficients. A $p$ value less than .05 was considered statistically significant.

\section{Ethics}

This study was approved by the International Review Board of the Catholic University Medical College of Korea (KC15EISI0103). All participants and their parents gave written informed consent.

\section{RESULTS}

\section{Demographic and clinical characteristics}

The characteristics of the participants are presented in Table 1. There was no significant difference between the IGC and IGD groups in age, sex, total education years of parents, or socioeconomic status. Mean daily time playing Internet games was significantly higher in the IGD group than the IGC group $(p=.000)$. The level of stress as measured by a stress scale was significantly higher in the IGD group than the IGC group $(p=.000)$. The IGD group had a significantly higher IGD score obtained by self-report than the IGC group $(p=.000)$. 
Secretion levels of orexin $A$ and oxytocin

To investigate whether expression levels of orexin A and oxytocin differed between the IGC and IGD groups, we analyzed the expression levels of the two proteins in the peripheral blood of each group. The expression levels of orexin A were significantly elevated in the IGD group $(596.05 \pm 124.87 \mathrm{pg} / \mathrm{ml})$ compared to the IGC group $(666.81 \pm 138.07 \mathrm{pg} / \mathrm{ml} ; z$ value $=2.405, p=.016$; Figure 1A). When we compared Internet gaming time with expression levels of orexin A in each group, Internet gaming time was not significantly correlated with expression changes of orexin A in either group (IGC: $r=-.054$, $p=.749$; IGD: $r=-.046, p=.806$; Figure $1 \mathrm{~B}$ ). On the other hand, although there was no statistically significant difference in the expression levels of oxytocin between the IGC and IGD groups, the levels of oxytocin in the IGD group $(134.72 \pm 52.46 \mathrm{pg} / \mathrm{ml})$ tended to be higher than in the IGC group $(110.84 \pm 43.98 \mathrm{pg} / \mathrm{ml} ; z$ value $=1.663$, $p=.096$; Figure 1C). Internet gaming time was not correlated with oxytocin expression in either group (IGC: $r=.015, p=.929$; IGD: $r=.215, p=.246$; Figure 1D).

\section{Secretion levels of hormones related to circadian rhythm and stress}

We measured secretion levels of melatonin and cortisol, which are known to affect circadian rhythm and stress, in the IGC and IGD groups. The expression levels of melatonin tended to be higher $(z$ value $=1.923, p=.055)$ in the
IGD group $(38.08 \pm 29.35 \mathrm{pg} / \mathrm{ml})$ than the IGC group $(65.12 \pm 58.68 \mathrm{pg} / \mathrm{ml}$; Figure 2A). Internet gaming time was not correlated with melatonin expression in either group (IGC: $r=-.191, p=.237 ;$ IGD: $r=-.117, p=.546$; Figure $2 \mathrm{~B}$ ). On the other hand, the expression levels of cortisol did not differ significantly between the IGC $(126.05 \pm 46.71 \mathrm{ng} / \mathrm{ml})$ and IGD $(136.48 \pm 73.49 \mathrm{ng} / \mathrm{ml})$ groups ( $z$ value $=0.676, p=.499$; Figure $2 \mathrm{C}$ ). Internet gaming time was not correlated with cortisol expression in either group (IGC: $r=.020, p=.904$; IGD: $r=.068$, $p=.717$; Figure 2D).

\section{Secretion levels of factors related to neurological disease}

To investigate whether neurological disease-related proteins (BDNF, sICAM-1, RANTES, and NCAM) were associated with Internet gaming addiction, we measured the expression levels of these proteins in the peripheral blood of IGC and IGD groups. The expression levels of BDNF did not differ between the IGC $(37.31 \pm 9.01 \mathrm{ng} / \mathrm{ml})$ and IGD $(35.81 \pm 9.95 \mathrm{ng} / \mathrm{ml})$ groups $(z$ value $=-1.164, p=.245$; Figure 3A). Apart from this, Internet gaming time was significantly negatively correlated with BNDF expression levels in the IGD group $(r=-.370, p=.041)$, whereas there was no correlation between Internet gaming time and BDNF expression in the IGC group $(r=-.004, p=.983$; Figure 3B). Expression levels of sICAM-1 did not differ between the IGC $(128.83 \pm 30.78 \mathrm{ng} / \mathrm{ml})$ and IGD $(159.64 \pm 72.47 \mathrm{ng} / \mathrm{ml})$ groups $(z$ value $=1.341, p=.180$; Figure $3 \mathrm{C}$ ). On the other hand, Internet gaming time and
A

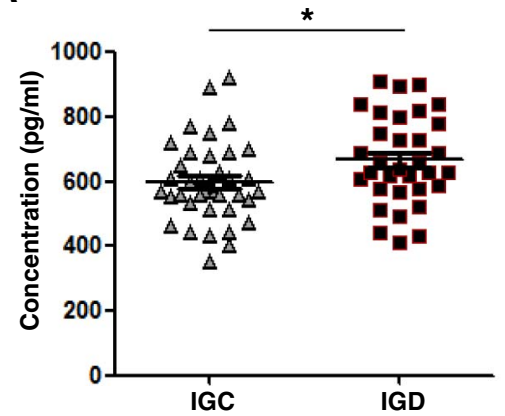

C

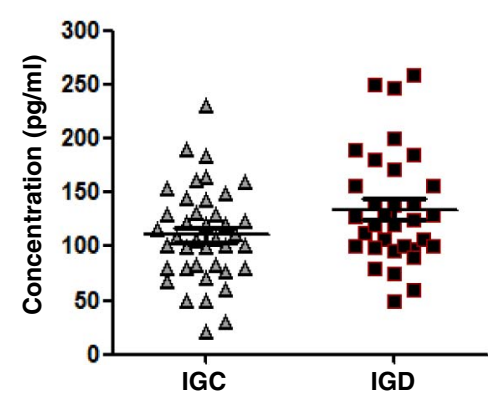

B

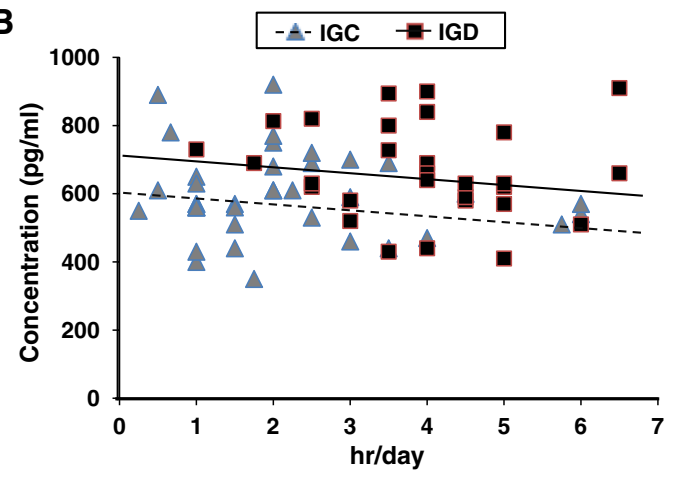

D

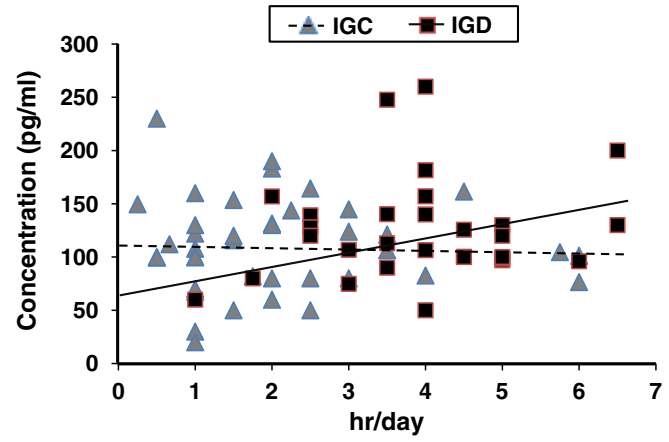

Figure 1. Expression levels of orexin A and oxytocin in the blood of adolescents. (A) Comparison of the expression levels of orexin A between Internet gaming control (IGC; $n=38$ ) and Internet gaming disorder (IGD; $n=33$ ) groups. (B) Correlation of orexin A expression with Internet gaming time within IGC and IGD groups. (C) Comparison of the expression levels of oxytocin between IGC $(n=40)$ and IGD $(n=33)$ groups. (D) Correlation of oxytocin expression with Internet gaming time within IGC and IGD groups. *Significant difference between IGC and IGD groups 

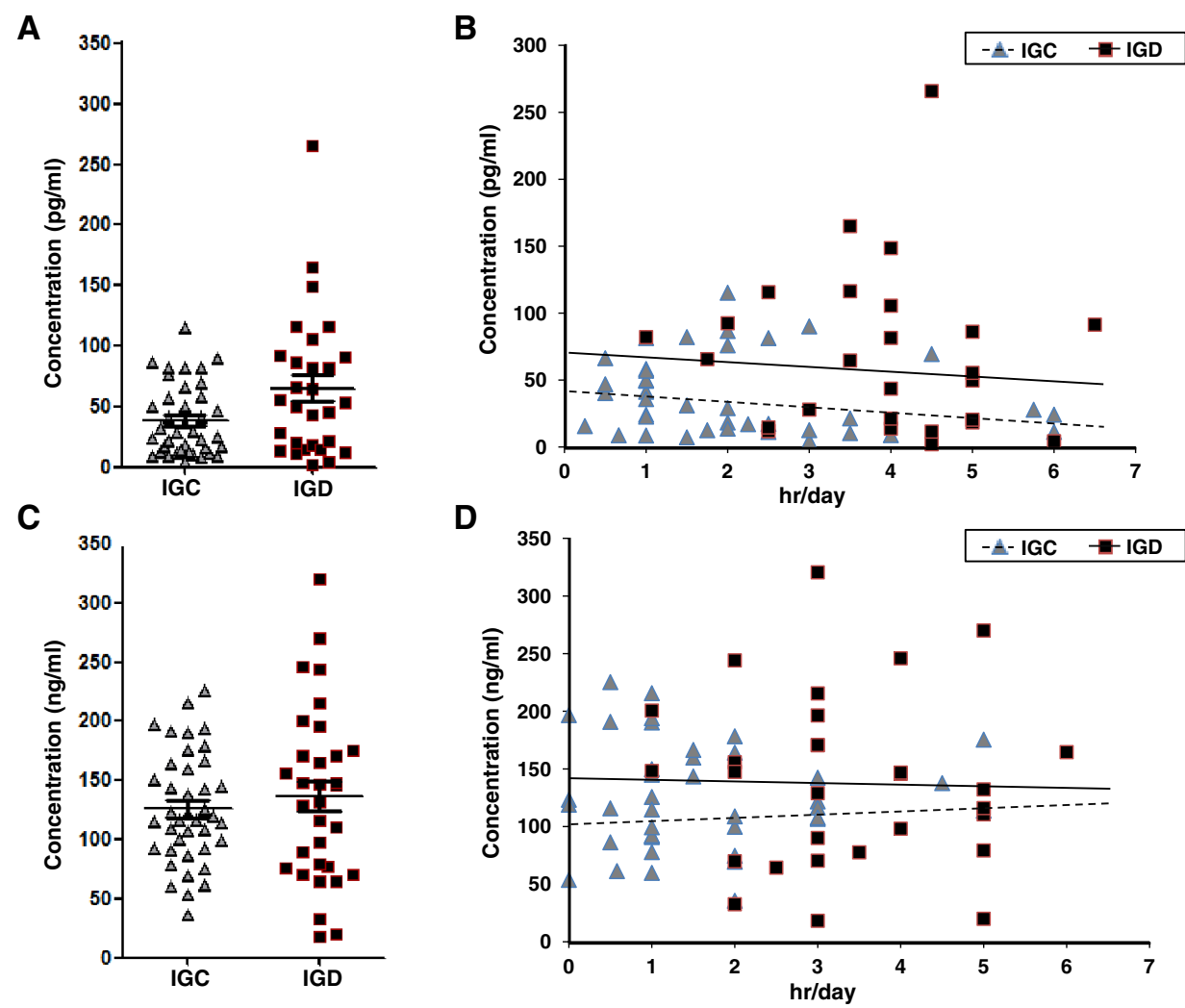

Figure 2. Expression levels of melatonin and cortisol in the blood of adolescents. (A) Comparison of the expression levels of melatonin between Internet gaming control (IGC; $n=39$ ) and Internet gaming disorder (IGD; $n=29$ ) groups. (B) Correlation of melatonin expression with Internet gaming time within the IGC and IGD groups. (C) Comparison of the expression levels of cortisol between IGC ( $n=40)$ and

IGD $(n=32)$ groups. (D) Correlation of cortisol expression with Internet gaming time within IGC and IGD groups

A

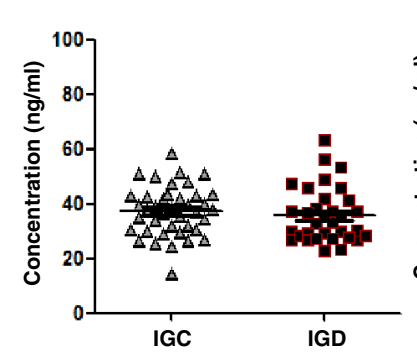

E

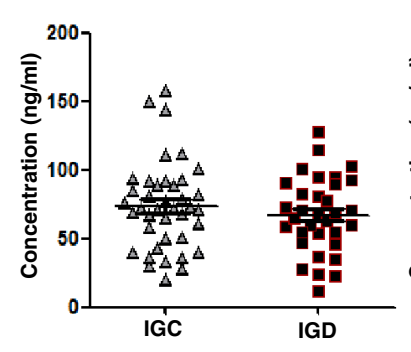

B

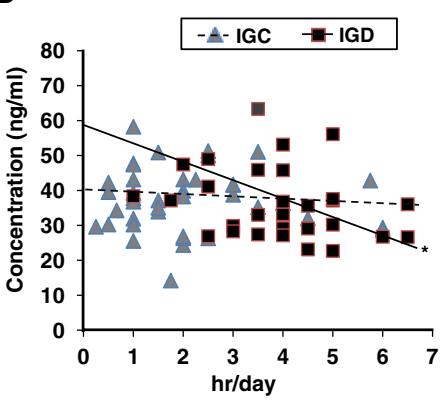

$\mathbf{F}$

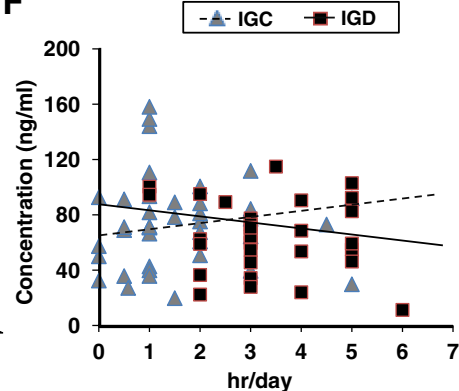

C

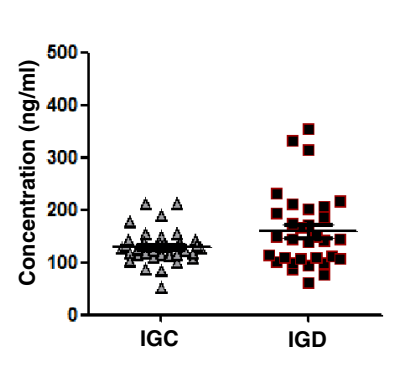

G

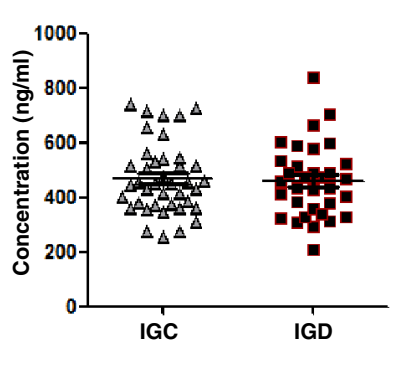

D

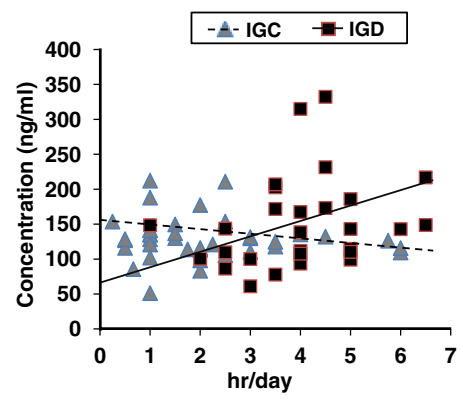

H

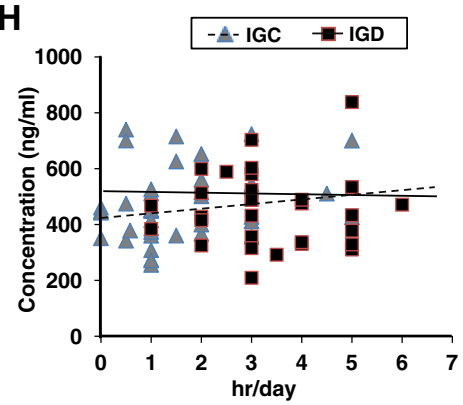

Figure 3. Expression levels of BDNF, sICAM-1, RANTES, and NCAM in the blood of adolescents. (A, C, E, and G) Comparison of expression levels of BDNF (IGC, $n=40$; IGD, $n=33$ ), sICAM-1 (IGC, $n=39$; IGD, $n=32$ ), RANTES (IGC, $n=40$; IGD, $n=32$ ), and NCAM (IGC, $n=40$; IGD, $n=32$ ) between IGC and IGD groups, respectively. (B, D, F, and H) Correlation of Internet gaming time with expression of BDNF, sICAM-1, RANTES, and NCAM, respectively, within each group. *Significant negative correlation of BDNF expression with Internet gaming time in the IGD group 
sICAM-1 concentration tended to be positively correlated in the IGD group $(r=.332, p=.073)$, whereas the IGC group did not show a tendency to correlation between Internet gaming time and sICAM-1 concentration $(r=-.142$, $p=.389$; Figure 3D). Expression levels of RANTES (IGC: $73.65 \pm 32.05 \mathrm{ng} / \mathrm{ml}$; IGD: $67.04 \pm 27.51 \mathrm{ng} / \mathrm{ml})$ and NCAM (IGC: $467.78 \pm 130.06 \mathrm{ng} / \mathrm{ml}$; IGD: $459.60 \pm 132.50 \mathrm{ng} / \mathrm{ml}$ ) did not differ between the IGC and IGD groups (RANTES: $z$ value $=-0.754, p=.451 ;$ NCAM: $z$ value $=-0.177$, $p=.859$; Figure $3 \mathrm{E}, \mathrm{G})$. Internet gaming time was not correlated with the change of RANTES (IGC: $r=.139$, $p=.392$; IGD: $r=-.117, p=.531$ ) or NCAM (IGC: $r=.178, p=.272$; IGD: $r=.011, p=.953)$ in either group (Figure 3F, H).

\section{DISCUSSION}

IGD is on the rise due to the rapid growth in Internet gaming technologies. Internet gaming addiction among adolescents, who experience important maturational rearrangements to hormonal and brain systems, is rapidly increasing, and this phenomenon can cause various social and psychological problems including anxiety and learning disability. To date, there exist few treatment protocols or biomarkers for diagnosis of IGD. In this study, we investigated changes in some of the proteins in the blood of adolescents with IGD and their biological implications for IGD diagnosis and treatment.

We found that secretion of orexin A was elevated in adolescents with IGD. Orexin A/hypocretin 1 is produced from orexin neurons in the lateral and dorsomedial hypothalamus and perifornical area. Recent studies have reported that orexin-induced signaling is associated with drugseeking behaviors through activation of orexin neurons in response to morphine, cocaine, or alcohol (Brown et al., 2016; Harris et al., 2005). Repeated drug-seeking behaviors by orexin-induced signaling can lead to drug addiction. Orexin neurons are also activated by a variety of stressors (James, Mahler, Moorman, \& Aston-Jones, 2017), while antagonists of orexin receptors reduce alcohol-seeking behaviors (Srinivasan et al., 2012). These findings indicate that orexin-induced signaling is involved in substance addiction. Similarly, IGD is also an addictive disorder, and individuals with IGD show addictive behaviors to Internet gaming (Petry et al., 2014). Carr and Kalivas (2006) have reported that orexin, as a gatekeeper of addiction, modulates motivated behaviors such as feeding and arousal. Since orexin A was elevated in adolescents with IGD, it is likely that orexin-induced signaling modulates IGD, a behavioral addiction.

Oxytocin is produced in the hypothalamus and emitted into the bloodstream. The released oxytocin functions as a hormone that affects peripheral targets through various mechanisms (Lee et al., 2016). Oxytocin in the brain also acts as a neuropeptide on increase of extracellular dopamine within the nucleus accumbens, pain, stress, and emotional regulation (Melis et al., 2007). In this study, oxytocin secretion tended to increase in the blood of adolescents with IGD. In contrast to our findings, some studies have reported that repeated administration of cocaine and alcohol decreases oxytocin in the plasma (Marchesi et al., 1997; Sarnyai et al., 1992). More recent studies have shown that the levels of plasma oxytocin are lower in individuals with schizophrenia than in normal individuals (Strauss et al., 2019), whereas levels of oxytocin are increased in depressed women, suggesting a dysregulated pattern of oxytocin release (Cyranowski et al., 2008). As a result, the expression pattern of oxytocin may vary according to the type of disease or addiction.

In this study, the expression of melatonin in adolescents with IGD tended to increase compared to normal adolescents. Melatonin modulates several physiological functions including circadian rhythms and inflammation (Hardeland, Cardinali, Brown, \& Pandi-Perumal, 2015). It is also a sleep hormone that is mainly released in the pineal gland in dark conditions and regulates sleep-wake cycles (Tarocco et al., 2019). In addition, melatonin antagonizes excitotoxicity caused by excessive glutamatergic stimulation in the brain, resulting in attenuation or prevention of neuroinflammation (Hardeland et al., 2015), and it plays a role in substance addictions including cocaine and alcohol (Takahashi et al., 2017; Vengeliene et al., 2015). A previous study found that male Wistar rats that receive melatonin at the end of the light phase showed reduction of relapse-like alcohol intake (Vengeliene et al., 2015). Another study reported that motivation for self-administration of cocaine in rats is reduced by melatonin (Takahashi et al., 2017). These findings suggest that melatonin can reduce the desire to seek out alcohol or cocaine, helping to prevent or attenuate substance addictions. In this study, melatonin expression in the blood tended to be increased in the IGD group, but there was no significant difference. Therefore, further studies are required to investigate expression level of melatonin in the blood of adolescents and adults with IGD to identify whether melatonin affects behavioral addiction.

BDNF has a pivotal role in modulation of neurotransmitter release, neuroprotection, and regulation of neuro-, synapto-, and gliogenesis (Chao, 2003; Foltran \& Diaz, 2016). In particular, it influences cognition and memory mechanisms by managing short- and long-lasting synaptic interactions (Kowianski et al., 2018). In this study, the expression levels of BDNF did not significantly differ between the IGC and IGD groups. This is consistent with a previous study that showed BDNF expression in a sample of male IGD individuals $(n=11)$ did not differ from a male normal control group $(n=10)$ who did not spend any time on Internet games (Geisel et al., 2013). Based on the results of our and Geisel et al.'s studies, there seems to be no difference in BDNF expression between IGD and control groups regardless of sex and age. On the other hand, studies in patients with PGD have reported that the expression levels of BDNF were enhanced in the blood of the PGD group (Angelucci et al., 2013; Choi et al., 2016; Geisel et al., 2012). These studies have predicted that an increase in BDNF in PGD patients may be a compensatory mechanism for normalizing dopaminergic transmission associated with midbrain dopamine release disrupted by the dysfunction of the reward system. Taken together, these findings in behavior-related addictions suggest that a BDNF-related mechanism in IGD may be different from that in PDG. Apart from this, in this study, comparing Internet gaming time and expression 
changes in BDNF in IGC or IGD groups, interestingly, BDNF expression changes, were negatively correlated with Internet gaming time in the IGD group. Considering that BDNF plays a pivotal role in brain development and synaptic plasticity and is important for maturational rearrangements in brain systems that happen during the adolescent period (Chao, 2003; Kowianski et al., 2018), our results suggest that reducing the initial Internet gaming time in adolescents diagnosed with IGD may attenuate the reduction of BDNF expression and help enhance BDNF function in the adolescent brain. However, based on our observation that there was no difference in BDNF expression between the IGC and IGD groups with a negative correlation between BDNF expression and Internet gaming time in the IGD group, further investigation of BDNFrelated mechanisms in IGD is needed.

In this study, changes in sICAM-1 expression in the blood tended to be positively correlated with Internet gaming time in adolescents with IGD. As a circulating form of ICAM-1, sICAM-1 is implicated in the development of various diseases. First, sICAM-1 is elevated in atherosclerosis, myocardial infarction, and cardiovascular diseases, indicating its potential as a biomarker for vascular inflammation (Hwang et al., 1997; Noutsias et al., 2003; Ridker et al., 1998). Second, increased sICAM-1 is related to the development of neurological diseases such as depression (Schaefer, Sarkar, Schwarz, \& Friebe, 2016). In addition, sICAM-1 is responsible for inflammatory processes in terms of neuropathology. Third, individuals with substance addictions to nicotine and alcohol exhibit elevated sICAM-1 in the peripheral blood (Bergmann et al., 1998; Rohde, Hennekens, \& Ridker, 1999). sICAM-1 is upregulated in people with obesity and it decreases as people lose weight (Ito et al., 2002). As a result, we can assume that increased sICAM-1 is a signal of neurological disease or addiction. sICAM-1 promotes activation of astrocytes or microglia in the brain and activation of lymphocytes in the blood, followed by activation of inflammation, resulting in negative effects in the body. Therefore, the tendency for positive correlation between sICAM-1 expression and Internet gaming time in adolescents with IGD in this study indicates that time spent on Internet gaming in adolescents with IGD may influence changes in the expression of sICAM-1.

Although we found that IGD was related to some proteins expressed in the blood, and that expression changes in BDNF and sICAM-1 were correlated with Internet gaming time in adolescents with IGD, this study had some limitations. First, the sample size was somewhat small. With a larger sample size, we could have obtained more robust findings of differences in protein expression between IGC and IGD groups. Second, blood collection was performed in the afternoon, and the blood samples were held at RT for more than $30 \mathrm{~min}$. Blood collection in the afternoon and delay before extracting serum may reduce the accuracy of the concentration determination in the blood when measuring hormones that are metabolized quickly. However, all blood was collected at the same conditions of collection time and coagulation time. Third, we did not control for factors such as sleeping duration, diet, and activity before blood tests among participants.
In summary, we investigated changes in hormones and neuropeptides associated with circadian rhythm, stress, and neurological diseases in adolescents with IGD. We found elevation of orexin $\mathrm{A}$ in the peripheral blood of adolescents with IGD compared to normal adolescents. We also found positive correlations between Internet gaming time and BDNF expression in adolescents with IGD. To our knowledge, this is the first study to identify expression change of orexin A and the correlation of BDNF expression change with Internet gaming time in the blood of adolescents with IGD. Our findings suggest that orexin A is correlated with IGD in adolescents and provide insight into an intervention to help adolescents at higher risk of IGD.

Funding sources: This work was supported by the National Research Foundation of Korea Grant funded by the Korean Government (NRF-2014M3C7A1062893). This funding source had no role in the design of this study and will not any role during its execution, analyses, interpretation of the data, or decision to submit results.

Authors' contribution: MRC wrote the first draft of the manuscript and measured protein concentrations. HC recruited the participants, evaluated them, performed statistical analysis, and interpreted the data. J-WC designed the study and recruited the participants. JHY helped to interpret the correlation of the value and IGD and reviewed the first draft of the manuscript. D-JK is responsible for the study concept and design and supervised all experimental procedures. All authors reviewed, revised, and approved the final version of the manuscript. MRC and HC contributed equally to this work.

Conflict of interest: The authors declare no conflict of interest.

\section{REFERENCES}

American Psychiatric Association. (2013). Diagnostic and statistical manual of mental disorders: DSM-5 (5th ed.). Washington, DC: American Psychiatric Association.

Angelucci, F., Martinotti, G., Gelfo, F., Righino, E., Conte, G., Caltagirone, C., Bria, P., \& Ricci, V. (2013). Enhanced BDNF serum levels in patients with severe pathological gambling. Addiction Biology, 18(4), 749-751. doi:10.1111/j.1369-1600. 2011.00411.x

Aydm, B., \& San, S. V. (2011). Internet addiction among adolescents: The role of self-esteem. Procedia - Social and Behavioral Sciences, 15, 3500-3505. doi:10.1016/j.sbspro.2011. 04.325

Baraczka, K., Nekam, K., Pozsonyi, T., Jakab, L., Szongoth, M., \& Sesztak, M. (2001). Concentration of soluble adhesion molecules (sVCAM-1, sICAM-1 and sL-selectin) in the cerebrospinal fluid and serum of patients with multiple sclerosis and systemic lupus erythematosus with central nervous involvement. Neuroimmunomodulation, 9(1), 49-54. doi:10.1159/ 000049007 
Barker, J. M., Torregrossa, M. M., \& Taylor, J. R. (2012). Low prefrontal PSA-NCAM confers risk for alcoholismrelated behavior. Nature Neuroscience, 15(10), 1356-1358. doi: 10.1038/nn.3194

Bergmann, S., Siekmeier, R., Mix, C., \& Jaross, W. (1998). Even moderate cigarette smoking influences the pattern of circulating monocytes and the concentration of SICAM-1. Respiration Physiology, 114(3), 269-275. doi:10.1016/S0034-5687(98) 00098-X

Bermudez, E. A., Rifai, N., Buring, J. E., Manson, J. E., \& Ridker, P. M. (2002). Relation between markers of systemic vascular inflammation and smoking in women. The American Journal of Cardiology, 89(9), 1117-1119. doi:10.1016/S0002-9149 (02)02284-1

Bonnavion, P., Jackson, A. C., Carter, M. E., \& de Lecea, L. (2015). Antagonistic interplay between hypocretin and leptin in the lateral hypothalamus regulates stress responses. Nature Communications, 6(1), 6266. doi:10.1038/ncomms 7266

Boyer, M., \& Dickerson, M. (2003). Attentional bias and addictive behaviour: Automaticity in a gambling-specific modified Stroop task. Addiction, 98(1), 61-70. doi:10.1046/j.13600443.2003.00219.x

Brown, R. M., Kim, A. K., Khoo, S. Y., Kim, J. H., Jupp, B., \& Lawrence, A. J. (2016). Orexin-1 receptor signalling in the prelimbic cortex and ventral tegmental area regulates cueinduced reinstatement of ethanol-seeking in iP rats. Addiction Biology, 21(3), 603-612. doi:10.1111/adb.12251

Cao, J. P., Wang, H. J., Li, L., \& Zhang, S. M. (2016). The effects of morphine treatment on the NCAM and its signaling in the MLDS of rats. Drug and Chemical Toxicology, 39(4), 418-423. doi:10.3109/01480545.2015.1137302

Carr, D., \& Kalivas, P. W. (2006). Orexin: A gatekeeper of addiction. Nature Medicine, 12(3), 274-276. doi:10.1038/ nm0306-274

Chao, M. V. (2003). Neurotrophins and their receptors: A convergence point for many signalling pathways. Nature Reviews Neuroscience, 4(4), 299-309. doi:10.1038/nrn1078

Choi, S. W., Shin, Y. C., Mok, J. Y., Kim, D. J., Choi, J. S., \& Suk-Hyun Hwang, S. (2016). Serum BDNF levels in patients with gambling disorder are associated with the severity of gambling disorder and Iowa Gambling Task indices. Journal of Behavioral Addictions, 5(1), 135-139. doi:10.1556/2006.5. 2016.010

Chun, J. W., Choi, J., Cho, H., Lee, S. K., \& Kim, D. J. (2015). Dysfunction of the frontolimbic region during swear word processing in young adolescents with Internet gaming disorder. Translstional Psychiatry, 5(8), e624. doi:10.1038/tp.2015.106

Cohen, S., Kamarck, T., \& Mermelstein, R. (1983). A global measure of perceived stress. Journal of Health and Social Behavior, 24(4), 385-396. doi:10.2307/2136404

Connor, K. M., Ceesay, P., Hutzelmann, J., Snavely, D., Krystal, A. D., Trivedi, M. H., Thase, M., Lines, C., Herring, W. J., \& Michelson, D. (2017). Phase II proof-of-concept trial of the orexin receptor antagonist filorexant (MK-6096) in patients with major depressive disorder. The International Journal of Neuropsychopharmacology, 20(8), 613-618. doi:10.1093/ ijnp/pyx033

Corominas, M., Roncero, C., Ribases, M., Castells, X., \& Casas, M. (2007). Brain-derived neurotrophic factor and its intracellular signaling pathways in cocaine addiction. Neuropsychobiology, 55(1), 2-13. doi:10.1159/000103570
Cyranowski, J. M., Hofkens, T. L., Frank, E., Seltman, H., Cai, H. M., \& Amico, J. A. (2008). Evidence of dysregulated peripheral oxytocin release among depressed women. Psychosomatic Medicine, 70(9), 967-975. doi:10.1097/PSY.0b013e318188ade4

Dalbudak, E., Evren, C., Aldemir, S., Coskun, K. S., Ugurlu, H., \& Yildirim, F. G. (2013). Relationship of internet addiction severity with depression, anxiety, and alexithymia, temperament and character in university students. Cyberpsychology, Behavior, and Social Networking, 16(4), 272-278. doi:10.1089/cyber. 2012.0390

Du, X., Liu, L., Yang, Y., Qi, X., Gao, P., Zhang, Y., Zhu, J., Du, G., Dai, S., Li, X., \& Zhang, Q. (2017). Diffusion tensor imaging of the structural integrity of white matter correlates with impulsivity in adolescents with Internet gaming disorder. Brain and Behavior, 7(8), e00753. doi:10.1002/brb3.753

Foltran, R. B., \& Diaz, S. L. (2016). BDNF isoforms: A round trip ticket between neurogenesis and serotonin? Journal of Neurochemistry, 138(2), 204-221. doi:10.1111/jnc.13658

Fumagalli, F., Di Pasquale, L., Caffino, L., Racagni, G., \& Riva, M. A. (2007). Repeated exposure to cocaine differently modulates BDNF mRNA and protein levels in rat striatum and prefrontal cortex. The European Journal of Neuroscience, 26(10), 2756-2763. doi:10.1111/j.1460-9568.2007.05918.x

Garcia-Marchena, N., Silva-Pena, D., Martin-Velasco, A. I., Villanua, M. A., Araos, P., Pedraz, M., Maza-Quiroga, R., Romero-Sanchiz, P., Rubio, G., Castilla-Ortega, E., Suarez, J., Rodriguez de Fonseca, F., \& Serrano, A. (2017). Decreased plasma concentrations of BDNF and IGF-1 in abstinent patients with alcohol use disorders. PLoS One, 12(11), e0187634. doi:10.1371/journal.pone.0187634

Geisel, O., Banas, R., Hellweg, R., \& Muller, C. A. (2012). Altered serum levels of brain-derived neurotrophic factor in patients with pathological gambling. European Addiction Research, 18(6), 297-301. doi:10.1159/000338281

Geisel, O., Banas, R., Schneider, M., Hellweg, R., \& Muller, C. A. (2013). Serum levels of brain-derived neurotrophic factor in patients with Internet use disorder. Psychiatry Research, 209(3), 525-528. doi:10.1016/j.psychres.2012.12.020

Han, D. H., Lee, Y. S., Yang, K. C., Kim, E. Y., Lyoo, I. K., \& Renshaw, P. F. (2007). Dopamine genes and reward dependence in adolescents with excessive Internet video game play. Journal of Addiction Medicine, 1(3), 133-138. doi:10.1097/ ADM.0b013e31811f465f

Hardeland, R., Cardinali, D. P., Brown, G. M., \& Pandi-Perumal, S. R. (2015). Melatonin and brain inflammaging. Progess in Neurobiology, 127-128, 46-63. doi:10.1016/j.pneurobio. 2015.02.001

Harris, G. C., Wimmer, M., \& Aston-Jones, G. (2005). A role for lateral hypothalamic orexin neurons in reward seeking. Nature, 437(7058), 556-559. doi:10.1038/nature04071

Hebert, S., Beland, R., Dionne-Fournelle, O., Crete, M., \& Lupien, S. J. (2005). Physiological stress response to video-game playing: The contribution of built-in music. Life Sciences, 76(20), 2371-2380.

Hwang, S. J., Ballantyne, C. M., Sharrett, A. R., Smith, L. C., Davis, C. E., Gotto, A. M., Jr., \& Boerwinkle, E. (1997). Circulating adhesion molecules VCAM-1, ICAM-1, and E-selectin in carotid atherosclerosis and incident coronary heart disease cases: The atherosclerosis risk in communities (ARIC) study. Circulation, 96(12), 4219-4225. doi:10.1161/01.CIR. 96.12.4219 
Ito, H., Ohshima, A., Inoue, M., Ohto, N., Nakasuga, K., Kaji, Y., Maruyama, T., \& Nishioka, K. (2002). Weight reduction decreases soluble cellular adhesion molecules in obese women. Clinical and Experimental Pharmacology \& Physiology, 29(5-6), 399-404. doi:10.1046/j.1440-1681.2002.03672.x

Izenwasser, S. (2005). Differential effects of psychoactive drugs in adolescents and adults. Critical Reviews in Neurobiology, 17(2), 51-68. doi:10.1615/CritRevNeurobiol.v17.i2.10

James, M. H., Mahler, S. V., Moorman, D. E., \& Aston-Jones, G. (2017). A decade of orexin/hypocretin and addiction: Where are we now? Current Topics in Behavioral Neurosciences, 33, 247-281. doi:10.1007/7854_2016_57

Johns, J. M., Caldwell, J. D., \& Pedersen, C. A. (1993). Acute cocaine treatment decreases oxytocin levels in the rat hippocampus. Neuropeptides, 24(3), 165-169. doi:10.1016/01434179(93)90081-K

Kang, K. D., Jung, T. W., Park, I. H., \& Han, D. H. (2018). Effects of equine-assisted activities and therapies on the affective network of adolescents with Internet gaming disorder. The Journal of Alternative and Complementary Medicine, 24(8), 841-849. doi:10.1089/acm.2017.0416

Kim, E. H., \& Kim, N. H. (2013). Comparison of stress level and HPA axis activity of internet game addiction vs. non-addiction in adolescents. Journal of Korean Biological Nursing Science, 15(4), 173-183. doi:10.7586/jkbns.2013.15.4.173

Kim, N., Sung, J. Y., Park, J. Y., Kong, I. D., Hughes, T. L., \& Kim, D. K. (2019). Association between Internet gaming addiction and leukocyte telomere length in Korean male adolescents. Social Science \& Medicine, 222, 84-90. doi:10.1016/j.socscimed.2018.12.026

Koob, G. F. (2003). Alcoholism: Allostasis and beyond. Alcoholism, Clinical and Experimental Research, 27(2), 232-243. doi:10.1097/01.ALC.0000057122.36127.C2

Kowianski, P., Lietzau, G., Czuba, E., Waskow, M., Steliga, A., \& Morys, J. (2018). BDNF: A key factor with multipotent impact on brain signaling and synaptic plasticity. Cellular and Molecular Neurobiology, 38(3), 579-593. doi:10.1007/ s10571-017-0510-4

Kraus, J., Oschmann, P., Engelhardt, B., Schiel, C., Hornig, C., Bauer, R., Kern, A., Traupe, H., \& Dorndorf, W. (1998). Soluble and cell surface ICAM-1 as markers for disease activity in multiple sclerosis. Acta Neurologica Scandinavica, 98(2), 102-109. doi:10.1111/j.1600-0404.1998.tb01727.x

Kuss, D. J., \& Griffiths, M. D. (2012). Internet gaming addiction: A systematic review of empirical research. International Journal of Mental Health and Addiction, 10(2), 278-296. doi:10.1007/ s11469-011-9318-5

Kuss, D. J., Griffiths, M. D., Karila, L., \& Billieux, J. (2014). Internet addiction: A systematic review of epidemiological research for the last decade. Current Pharmaceutical Design, 20(25), 4026-4052. doi:10.2174/13816128113199990617

Lee, M., Cho, H., Jung, S. H., Yim, S. H., Cho, S. M., Chun, J. W., Paik, S. H., Park, Y. E., Cheon, D. H., Lee, J. E., Choi, J. S., Kim, D. J., \& Chung, Y. J. (2018). Circulating microRNA expression levels associated with Internet gaming disorder. Frontiers in Psychiatry, 9, 81. doi:10.3389/fpsyt.2018.00081

Lee, M. R., Rohn, M. C., Tanda, G., \& Leggio, L. (2016). Targeting the oxytocin system to treat addictive disorders: Rationale and progress to date. CNS Drugs, 30(2), 109-123. doi:10.1007/s40263-016-0313-z
Lubman, D. I., Cheetham, A., \& Yucel, M. (2015). Cannabis and adolescent brain development. Pharmacology \& Therapeutics, 148, 1-16. doi:10.1016/j.pharmthera.2014.11.009

Lynn, J. M. (1970). Intravenous alcohol infusion for premature labor. The Journal of the American Osteopathic Association, 70(2), 167-170. Retrieved from https://jaoa.org/article.aspx? articleid $=2096351$

Marchesi, C., Chiodera, P., Brusamonti, E., Volpi, R., \& Coiro, V. (1997). Abnormal plasma oxytocin and beta-endorphin levels in alcoholics after short and long term abstinence. Progress in Neuropsychopharmacology and Biological Psychiatry, 21(5), 797-807. doi:10.1016/S0278-5846(97)00080-8

Melis, M. R., Melis, T., Cocco, C., Succu, S., Sanna, F., Pillolla, G., Boi, A., Ferri, G. L., \& Argiolas, A. (2007). Oxytocin injected into the ventral tegmental area induces penile erection and increases extracellular dopamine in the nucleus accumbens and paraventricular nucleus of the hypothalamus of male rats. The European Journal of Neuroscience, 26(4), 1026-1035. doi:10.1111/j.1460-9568.2007.05721.x

Mikolajczyk, T. P., Nosalski, R., Szczepaniak, P., Budzyn, K., Osmenda, G., Skiba, D., Sagan, A., Wu, J., Vinh, A., Marvar, P. J., Guzik, B., Podolec, J., Drummond, G., Lob, H. E., Harrison, D. G., \& Guzik, T. J. (2016). Role of chemokine RANTES in the regulation of perivascular inflammation, T-cell accumulation, and vascular dysfunction in hypertension. FASEB Journal, 30(5), 1987-1999. doi:10.1096/fj.201500088R

Mohs, A., Kuttkat, N., Reissing, J., Zimmermann, H. W., Sonntag, R., Proudfoot, A., Youssef, S. A., de Bruin, A., Cubero, F. J., \& Trautwein, C. (2017). Functional role of CCL5/RANTES for HCC progression during chronic liver disease. Journal of Hepatology, 66(4), 743-753. doi:10.1016/j.jhep.2016.12.011

Moreno, M. A., Jelenchick, L., Cox, E., Young, H., \& Christakis, D. A. (2011). Problematic Internet use among US youth: A systematic review. Archives of Pediatrics \& Adolescent Medicine, 165(9), 797-805. doi:10.1001/archpediatrics. 2011.58

Müller, K. W., Glaesmer, H., Brähler, E., Woelfling, K., \& Beutel, M. E. (2014). Prevalence of Internet addiction in the general population: Results from a German population-based survey. Behaviour \& Information Technology, 33(7), 757-766. doi:10.1080/0144929X.2013.810778

Noutsias, M., Hohmann, C., Pauschinger, M., Schwimmbeck, P. L., Ostermann, K., Rode, U., Yacoub, M. H., Kuhl, U., \& Schultheiss, H. P. (2003). sICAM-1 correlates with myocardial ICAM-1 expression in dilated cardiomyopathy. International Journal of Cardiology, 91(2-3), 153-161. doi:10.1016/ S0167-5273(03)00033-0

O’Halloran, E. B., Curtis, B. J., Afshar, M., Chen, M. M., Kovacs, E. J., \& Burnham, E. L. (2016). Alveolar macrophage inflammatory mediator expression is elevated in the setting of alcohol use disorders. Alcohol, 50, 43-50. doi:10.1016/j.alcohol.2015. 11.003

Onaolapo, O. J., \& Onaolapo, A. Y. (2018). Melatonin in drug addiction and addiction management: Exploring an evolving multidimensional relationship. World Journal of Psychiatry, 8(2), 64-74. doi:10.5498/wjp.v8.i2.64

Ornell, F., Hansen, F., Schuch, F. B., Pezzini Rebelatto, F., Tavares, A. L., Scherer, J. N., Valerio, A. G., Pechansky, F., Paim Kessler, F. H., \& von Diemen, L. (2018). Brain-derived neurotrophic factor in substance use disorders: A systematic review 
and meta-analysis. Drug and Alcohol Dependence, 193, 91-103. doi:10.1016/j.drugalcdep.2018.08.036

Pan, N., Yang, Y., Du, X., Qi, X., Du, G., Zhang, Y., Li, X., \& Zhang, Q. (2018). Brain structures associated with Internet addiction tendency in adolescent online game players. Frontiers in Psychiatry, 9, 67. doi:10.3389/fpsyt.2018.00067

Park, J., \& Seo, Y. S. (2010). Validation of the perceived stress scale (PSS) on samples of Korean university students. Korean Journal of Psychology: General, 29(3), 611-629. Retrieved from http://www.dbpia.co.kr/journal/articleDetail? nodeId=NODE06368163

Pereira, J., Saez, C. G., Pallavicini, J., Panes, O., Pereira-Flores, K., Cabreras, M. J., Massardo, T., \& Mezzano, D. (2011). Platelet activation in chronic cocaine users: Effect of short term abstinence. Platelets, 22(8), 596-601. doi:10.3109/09537104. 2011.578181

Petry, N. M., Rehbein, F., Gentile, D. A., Lemmens, J. S., Rumpf, H. J., Mossle, T., Bischof, G., Tao, R., Fung, D. S., Borges, G., Auriacombe, M., Gonzalez Ibanez, A., Tam, P., \& O’Brien, C. P. (2014). An international consensus for assessing Internet gaming disorder using the new DSM-5 approach. Addiction, 109(9), 1399-1406. doi:10.1111/add.12457

Qi, X., Du, X., Yang, Y., Du, G., Gao, P., Zhang, Y., Qin, W., Li, X., \& Zhang, Q. (2015). Decreased modulation by the risk level on the brain activation during decision making in adolescents with Internet gaming disorder. Frontiers in Behavioral Neuroscience, 9, 296. doi:10.3389/fnbeh.2015.00296

Ridker, P. M., Hennekens, C. H., Roitman-Johnson, B., Stampfer, M. J., \& Allen, J. (1998). Plasma concentration of soluble intercellular adhesion molecule 1 and risks of future myocardial infarction in apparently healthy men. Lancet, 351(9096), 88-92. doi:10.1016/S0140-6736(97)09032-6

Rohde, L. E., Hennekens, C. H., \& Ridker, P. M. (1999). Crosssectional study of soluble intercellular adhesion molecule-1 and cardiovascular risk factors in apparently healthy men. Arteriosclerosis, Thrombosis, and Vascular Biology, 19(7), 1595-1599. doi:10.1161/01.ATV.19.7.1595

Sacanella, E., Estruch, R., Badia, E., Fernandez-Sola, J., Nicolas, J. M., \& Urbano-Marquez, A. (1999). Chronic alcohol consumption increases serum levels of circulating endothelial cell/ leucocyte adhesion molecules E-selectin and ICAM-1. Alcohol Alcohol, 34(5), 678-684. doi:10.1093/alcalc/34.5.678

Sagrillo-Fagundes, L., Bienvenue-Pariseault, J., \& Vaillancourt, C. (2019). Melatonin: The smart molecule that differentially modulates autophagy in tumor and normal placental cells. PLoS One, 14(1), e0202458. doi:10.1371/journal.pone.0202458

Sakurai, T., Amemiya, A., Ishii, M., Matsuzaki, I., Chemelli, R. M., Tanaka, H., Williams, S. C., Richardson, J. A., Kozlowski, G. P., Wilson, S., Arch, J. R., Buckingham, R. E., Haynes, A. C., Carr, S. A., Annan, R. S., McNulty, D. E., Liu, W. S., Terrett, J. A., Elshourbagy, N. A., Bergsma, D. J., \& Yanagisawa, M. (1998). Orexins and orexin receptors: A family of hypothalamic neuropeptides and $G$ proteincoupled receptors that regulate feeding behavior. Cell, 92(4), 573-585. doi:10.1016/S0092-8674(00)80949-6

Sarnyai, Z., \& Kovacs, G. L. (1994). Role of oxytocin in the neuroadaptation to drugs of abuse. Psychoneuroendocrinology, 19(1), 85-117. doi:10.1016/0306-4530(94)90062-0

Sarnyai, Z., Vecsernyes, M., Laczi, F., Biro, E., Szabo, G., \& Kovacs, G. L. (1992). Effects of cocaine on the contents of neurohypophyseal hormones in the plasma and in different brain structures in rats. Neuropeptides, 23(1), 27-31. doi:10.1016/0143-4179(92)90006-I

Schaefer, M., Sarkar, S., Schwarz, M., \& Friebe, A. (2016). Soluble intracellular adhesion molecule-1 in patients with unipolar or bipolar affective disorders: Results from a pilot trial. Neuropsychobiology, 74(1), 8-14. doi:10.1159/000446919

Srinivasan, S., Simms, J. A., Nielsen, C. K., Lieske, S. P., BitoOnon, J. J., Yi, H., Hopf, F. W., Bonci, A., \& Bartlett, S. E. (2012). The dual orexin/hypocretin receptor antagonist, almorexant, in the ventral tegmental area attenuates ethanol selfadministration. PLoS One, 7(9), e44726. doi:10.1371/ journal.pone. 0044726

Steiner, N., Rossetti, C., Sakurai, T., Yanagisawa, M., de Lecea, L., Magistretti, P. J., Halfon, O., \& Boutrel, B. (2018). Hypocretin/ orexin deficiency decreases cocaine abuse liability. Neuropharmacology, 133, 395-403. doi:10.1016/j.neuropharm. 2018.02.010

Strauss, G. P., Chapman, H. C., Keller, W. R., Koenig, J. I., Gold, J. M., Carpenter, W. T., \& Buchanan, R. W. (2019). Endogenous oxytocin levels are associated with impaired social cognition and neurocognition in schizophrenia. Journal of Psychiatric Research, 112, 38-43. doi:10.1016/j.jpsychires. 2019.02.017

Sweet, D. C., Levine, A. S., Billington, C. J., \& Kotz, C. M. (1999). Feeding response to central orexins. Brain Research, 821(2), 535-538. doi:10.1016/S0006-8993(99)01136-1

Takahashi, T. T., Vengeliene, V., \& Spanagel, R. (2017). Melatonin reduces motivation for cocaine self-administration and prevents relapse-like behavior in rats. Psychopharmacology (Berl), 234(11), 1741-1748. doi:10.1007/s00213-017-4576-y

Tarocco, A., Caroccia, N., Morciano, G., Wieckowski, M. R., Ancora, G., Garani, G., \& Pinton, P. (2019). Melatonin as a master regulator of cell death and inflammation: Molecular mechanisms and clinical implications for newborn care. Cell Death \& Disease, 10(4), 317. doi:10.1038/s41419-019-1556-7

Torres-Rodriguez, A., Griffiths, M. D., Carbonell, X., \& Oberst, U. (2018). Internet gaming disorder in adolescence: Psychological characteristics of a clinical sample. Journal of Behavioral Addictions, 7(3), 707-718. doi:10.1556/2006.7.2018.75

Veillard, N. R., Kwak, B., Pelli, G., Mulhaupt, F., James, R. W., Proudfoot, A. E., \& Mach, F. (2004). Antagonism of RANTES receptors reduces atherosclerotic plaque formation in mice. Circulation Research, 94(2), 253-261. doi:10.1161/01.RES. 0000109793.17591.4E

Vengeliene, V., Noori, H. R., \& Spanagel, R. (2015). Activation of melatonin receptors reduces relapse-like alcohol consumption. Neuropsychopharmacology, 40(13), 2897-2906. doi:10.1038/ npp.2015.143

Wang, H. R., Cho, H., \& Kim, D. J. (2018). Prevalence and correlates of comorbid depression in a nonclinical online sample with DSM-5 Internet gaming disorder. Journal of Affective Disorders, 226, 1-5. doi:10.1016/j.jad.2017.08.005

Widyanto, L., \& Griffiths, M. (2006). 'Internet Addiction': A critical review. International Journal of Mental Health and Addiction, 4(1), 31-51. doi:10.1007/s11469-006-9009-9

World Health Organization [WHO]. (2018). International classification of diseases: ICD-11 for mortality and morbidity statistics. 6C51 gaming disorder. Retrieved from https://icd.who.int/ browse11/1-m/en\#/http://id.who.int/icd/entity/1448597234

Xu, Z., Wang, C., Dong, X., Hu, T., Wang, L., Zhao, W., Zhu, S., Li, G., Hu, Y., Gao, Q., Wan, J., Liu, Z., \& Sun, J. (2019). 
Chronic alcohol exposure induced gut microbiota dysbiosis and its correlations with neuropsychic behaviors and brain BDNF/Gabra1 changes in mice. Biofactors, 45(2), 187-199. doi:10.1002/biof.1469

Yiallouris, A., Tsioutis, C., Agapidaki, E., Zafeiri, M., Agouridis, A. P., Ntourakis, D., \& Johnson, E. O. (2019). Adrenal aging and its implications on stress responsiveness in humans. Frontiers in Endocrinology (Lausanne), 10, 54. doi:10.3389/ fendo.2019.00054
Young, K. S. (2004). Internet addiction: A new clinical phenomenon and its consequences. American Behavioral Scientist, 48(4), 402-415. doi:10.1177/0002764204270278

Zhou, Y., Guo, W., Zhu, Z., Hu, Y., Wang, Y., Zhang, X., Wang, W., Du, N., Song, T., Yang, K., Guan, Z., Wang, Y., \& Guo, A. (2018). Macrophage migration inhibitory factor facilitates production of CCL5 in astrocytes following rat spinal cord injury. Journal of Neuroinflammation, 15(1), 253. doi:10.1186/s12974018-1297-z 